\title{
Activation of BNIP3-mediated mitophagy protects against renal ischemia-reperfusion injury
}

\author{
Chengyuan Tang1, Hailong Han², Zhiwen Liu', Yuxue Liu', Lijun Yin', Juan Cai', Liyu He', Yu Liu', Guochun Chen', \\ Zhuohua Zhang', Xiao-Ming Yin ${ }^{3}$ and Zheng Dong ${ }^{1}$
}

\begin{abstract}
Acute kidney injury (AKI) is a syndrome of abrupt loss of renal functions. The underlying pathological mechanisms of AKI remain largely unknown. BCL2-interacting protein 3 (BNIP3) has dual functions of regulating cell death and mitophagy, but its pathophysiological role in AKI remains unclear. Here, we demonstrated an increase of BNIP3 expression in cultured renal proximal tubular epithelial cells following oxygen-glucose deprivation-reperfusion (OGDR) and in renal tubules after renal ischemia-reperfusion (IR)-induced injury in mice. Functionally, silencing Bnip3 by specific short hairpin RNAs in cultured renal tubular cells reduced OGD-R-induced mitophagy, and potentiated OGD-Rinduced cell death. In vivo, Bnip3 knockout worsened renal IR injury, as manifested by more severe renal dysfunction and tissue injury. We further showed that Bnip3 knockout reduced mitophagy, which resulted in the accumulation of damaged mitochondria, increased production of reactive oxygen species, and enhanced cell death and inflammatory response in kidneys following renal IR. Taken together, these findings suggest that BNIP3-mediated mitophagy has a critical role in mitochondrial quality control and tubular cell survival during AKI.
\end{abstract}

\section{Introduction}

Acute kidney injury (AKI) is a clinical syndrome of abrupt loss of renal functions that is pathologically featured by sublethal and lethal injury of renal tubules. The clinical risk factors for AKI mainly include renal ischemia-reperfusion (IR), nephrotoxins, and sepsis. AKI has poor short- and long-term outcome. Besides its high short-term mortality, AKI has been recently recognized as an important risk factor for the development of chronic kidney disease $(\mathrm{CKD})^{1,2}$. Recent experimental studies have demonstrated that the pathogenesis of AKI is complex, involving tubular, microvascular, and inflammatory

\footnotetext{
Correspondence: Chengyuan Tang (tangchengyuan@csu.edu.cn) or Zheng Dong (zdong@csu.edu.cn)

${ }^{1}$ Department of Nephrology, The Second Xiangya Hospital, Central South University, Hunan Key Laboratory of Kidney Disease and Blood Purification, Changsha, Hunan, China

${ }^{2}$ Institute of Molecular Precision Medicine, Xiangya Hospital and Center for Medical Genetics, Central South University, Changsha, Hunan, China Full list of author information is available at the end of the article. These authors contributed equally: Chengyuan Tang, Hailong Han Edited by G. M. Fimia
}

mechanisms, diverse signal pathways, and molecules ${ }^{3}$. Of note, studies have highlighted a pivotal role of mitochondrial dysfunction in AKI development and progress ${ }^{4-9}$. Targeting mitochondrial protection and timely removal of damaged mitochondria have been proposed as promising strategies to prevent, treat AKI, and impede AKI-CKD transition $^{10-13}$.

Mitophagy is a specific form of autophagy that selectively degrades mitochondria. As a critical component of mitochondrial quality control, mitophagy targets damaged or dysfunctional mitochondria for prompt elimination, thereby preventing excessive reactive oxygen species (ROS) production, and release of mitochondrial pro-apoptotic factors and damage-associated molecular patterns (DAMPs) that may promote pathological inflammatory responses ${ }^{6,14}$. Mechanistically, mitophagy requires a coordinative induction of autophagy and mitochondrial priming for autophagic recognition. Thus, two mitochondrial priming mechanisms have been proposed, which rely on PTEN-induced kinase 1 (PINK1)parkin RBR E3 ubiquitin protein ligase (PARK2) pathway

\section{(c) The Author(s) 2019}

(c) (i) Open Access This article is licensed under a Creative Commons Attribution 4.0 International License, which permits use, sharing, adaptation, distribution and reproduction cc) in any medium or format, as long as you give appropriate credit to the original author(s) and the source, provide a link to the Creative Commons license, and indicate if changes were made. The images or other third party material in this article are included in the article's Creative Commons license, unless indicated otherwise in a credit line to the material. If material is not included in the article's Creative Commons license and your intended use is not permitted by statutory regulation or exceeds the permitted use, you will need to obtain permission directly from the copyright holder. To view a copy of this license, visit http://creativecommons.org/licenses/by/4.0/. 
and mitophagy receptors BCL2-interacting protein 3 like (BNIP3L/NIX), BCL2-interacting protein 3 (BNIP3), or FUN14 domain containing $1^{15,16}$. Recent evidence from other studies and our study suggests an involvement of mitophagy in AKI pathogenesis ${ }^{17-21}$. However, its precise roles and regulation in AKI remain not fully understood.

BNIP3 is a Bcl-2 family protein with an atypical BH3 domain that primarily localizes in mitochondrial out membrane. BNIP3 was firstly identified as a pro-apoptotic protein. Its induction has been shown to sensitize BAX (BCL2-associated X, apoptosis regulator) and BAK (BCL2 antagonist/killer 1) insertion and activation in the mitochondria, a key step for mitochondrial outer membrane permeabilization that results in the release of proapoptotic factors in the inter-membrane mitochondrial space into the cytosol to initiate apoptosis cascade ${ }^{22,23}$. On the other hand, recent studies suggest that BNIP3 is also a mitophagy receptor, and plays pro-survival roles in some pathological conditions ${ }^{24-27}$. In kidney, emerging evidence indicated that BNIP3 might have a role in the regulation of mitophagy in cultured renal proximal tubular cells (RPTCs) in response to oxidative stress and hypoxia $^{20}$, but its precise role in AKI pathogenesis remains largely unknown. In the present study, we have investigated the role of BNIP3 in the development of ischemic AKI by using cultured RPTCs subjected to oxygen-glucose deprivation and reoxygenation (OGD-R) and a mouse model of renal IR injury as model systems. The results support that BNIP3 is an important mediator of mitophagy in renal tubular cells, and BNIP3-dependent mitophagy plays a critical role in mitochondrial quality control for tubular cell viability and function in AKI.

\section{Results}

Suppression of Bnip3 expression sensitizes BUPMT cells to OGD-R injury in vitro

We first evaluated the expression of BNIP3 in Boston University mouse proximal tubule (BUMPT) cells that were subjected to OGD-R to mimic in vivo IR. Immunoblotting analysis showed a dramatic increase of BNIP3 in BUMPT cells after OGD-R (Fig. 1b, c). Specific Bnip3short hairpin RNAs (shRNAs) dramatically reduced BNIP3 expression (Fig. 1a, c). Terminal deoxynucleotidyl transferase-mediated dUTP nick-end labeling (TUNEL) assay demonstrated that Bnip3 knockdown (KD) showed minimal effect on TUNEL labeling under controlled condition, but dramatically increased the number of TUNEL-positive cells after OGD-R (Fig. 1d, e). Consistently, immunoblot of active/cleaved caspase-3 demonstrated that Bnip3 KD cells had a significantly higher level of activated caspase-3 than wild-type (WT) cells after OGD-R (Fig. 1f, g). Taken together, these findings support that BNIP3 silencing increases the sensitivity of proximal tubular cells to OGD-R-induced apoptosis, suggesting a pro-survival role of BNIP3 in these cells.

\section{Suppression of Bnip3 expression reduces OGD-R-induced mitophagy in BUPMT cells}

BNIP3 regulates both cell death and mitophagy. Our above results showed pro-survival functions of BNIP3 in BUPMT cells (Fig. 1d-g). We therefore focused on its potential role in the regulation of mitophagy. Immunoblotting analysis showed a remarkable increase of autophagosome marker microtubule-associated protein 1 light chain $3 \beta$ (MAP1LC3B/LC3B-II) and a decrease of specific autophagy substrate sequestosome 1 (SQSTM1) in BUPMT cells following OGD-R, indicating autophagy activation (Fig. 2a-c). Moreover, the alterations in LC3BII and SQSTM1 were associated with a marked reduction of mitochondrial membrane protein translocase of inner mitochondrial membrane 23 (TIMM23) and translocase of outer mitochondrial membrane 20 (TOMM20) (Fig. 2a, $\mathrm{d}$, e), suggesting an induction of mitophagy. Notably, Bnip3 KD resulted in less LC3B-II accumulation, and partially reduced the degradation of SQSTM1 as well as TIMM23 and TOMM20 in BUMPT cells following OGD$\mathrm{R}$ (Fig. 2a-e). Collectively, these findings suggested an important role of BNIP3 in the regulation of mitophagy in BUPMT cells during OGD-R. To further verify the promitophagy function of BNIP3, we evaluated mitophagosome formation by assessing the colocalization of mitochondria and autophagosomes. As shown in Fig. 2f, under controlled condition, both WT and Bnip3 KD cells had very few green fluorescent protein (GFP)-LC3B puncta, indicating a low level of autophagy. In the setting of OGD-R, an increase of GFP-LC3B puncta occurred in both WT and Bnip3 KD cells, and partial GFP-LC3B puncta colocalized with the mitochondria (Fig. 2f), suggesting the formation of mitophagosomes. Notably, quantification analysis showed that OGD-R induced much less autophagosome and mitophagosome formation in Bnip3-KD cells than in WT cells, suggesting an inhibitory effect of Bnip3 KD on mitophagy (Fig. 2g, f). Taken together, these results suggest a pro-mitophagy role of BNIP3 in RPTCs.

\section{Bnip3 deficiency exacerbates renal IR-induced kidney injury in vivo}

We then determined the role of BNIP3 in the pathogenesis of ischemic AKI in vivo. We first examined the expression of BNIP3 in kidney tissues of mouse models of ischemic AKI that was induced by $30 \mathrm{~min}$ of bilateral kidney ischemia, followed by $48 \mathrm{~h}$ of reperfusion. Immunohistochemical analysis showed that BNIP3 was dramatically induced in cortical renal proximal tubules of ischemic mice (Fig. 3a). Immunoblotting analysis confirmed the induction of BNIP3 in kidney tissues 


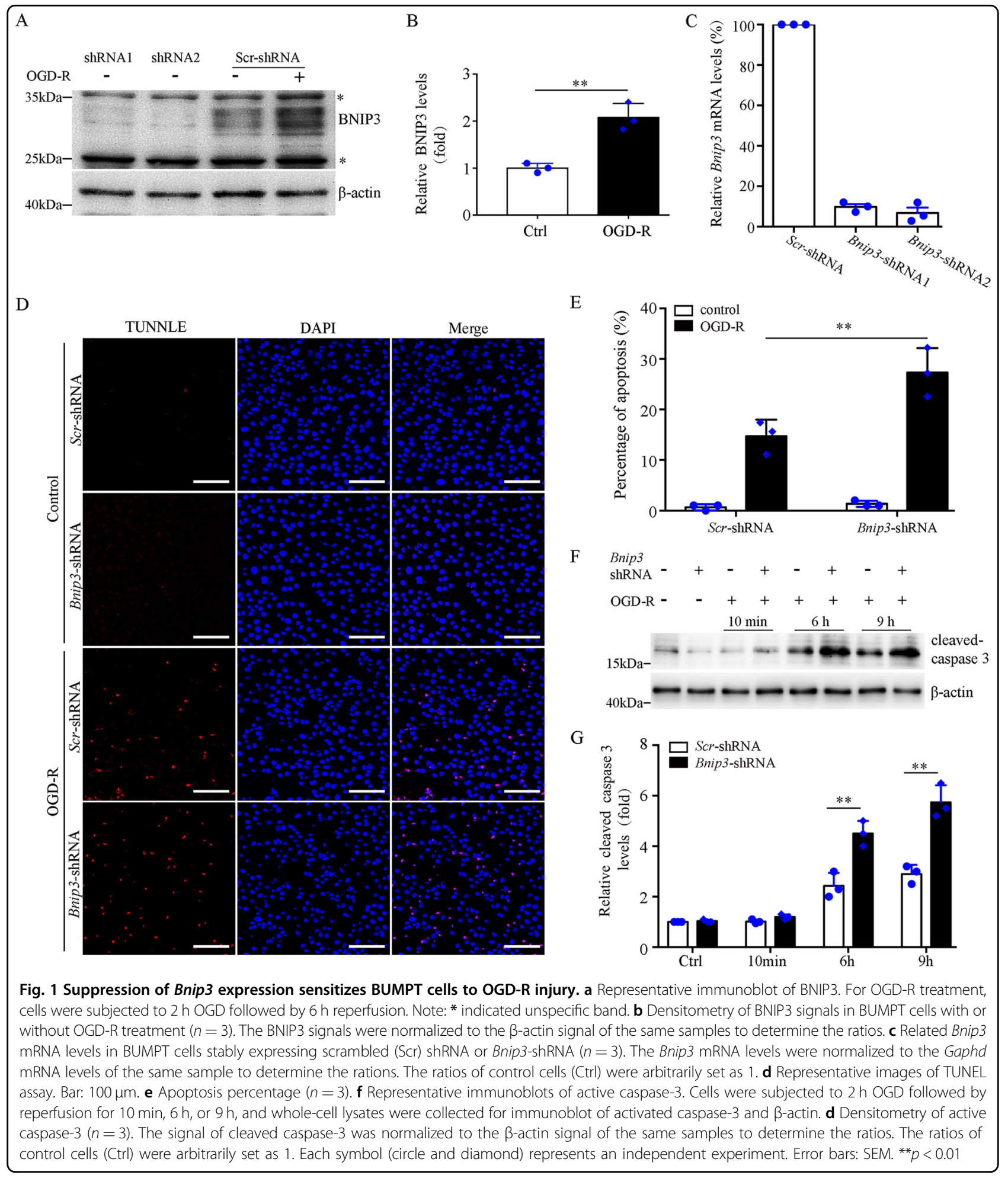

following renal IR (Fig. 3b, c). The above finding provided in vivo evidence for the induction of BNIP3 in RPTCs in ischemic AKI. To verify the role of BNIP3 in the pathogenesis of ischemic AKI, Bnip3-knockout (KO) mice were applied (Fig. 3a, b). Histological examination by hematoxylin and eosin (HE) staining showed that both Bnip3-KO mice and their WT control littermates had normal kidney structure under physiological condition, but showed obvious tubular injury in the setting of renal IR (Fig. 3d). Quantitative analysis showed that KO 


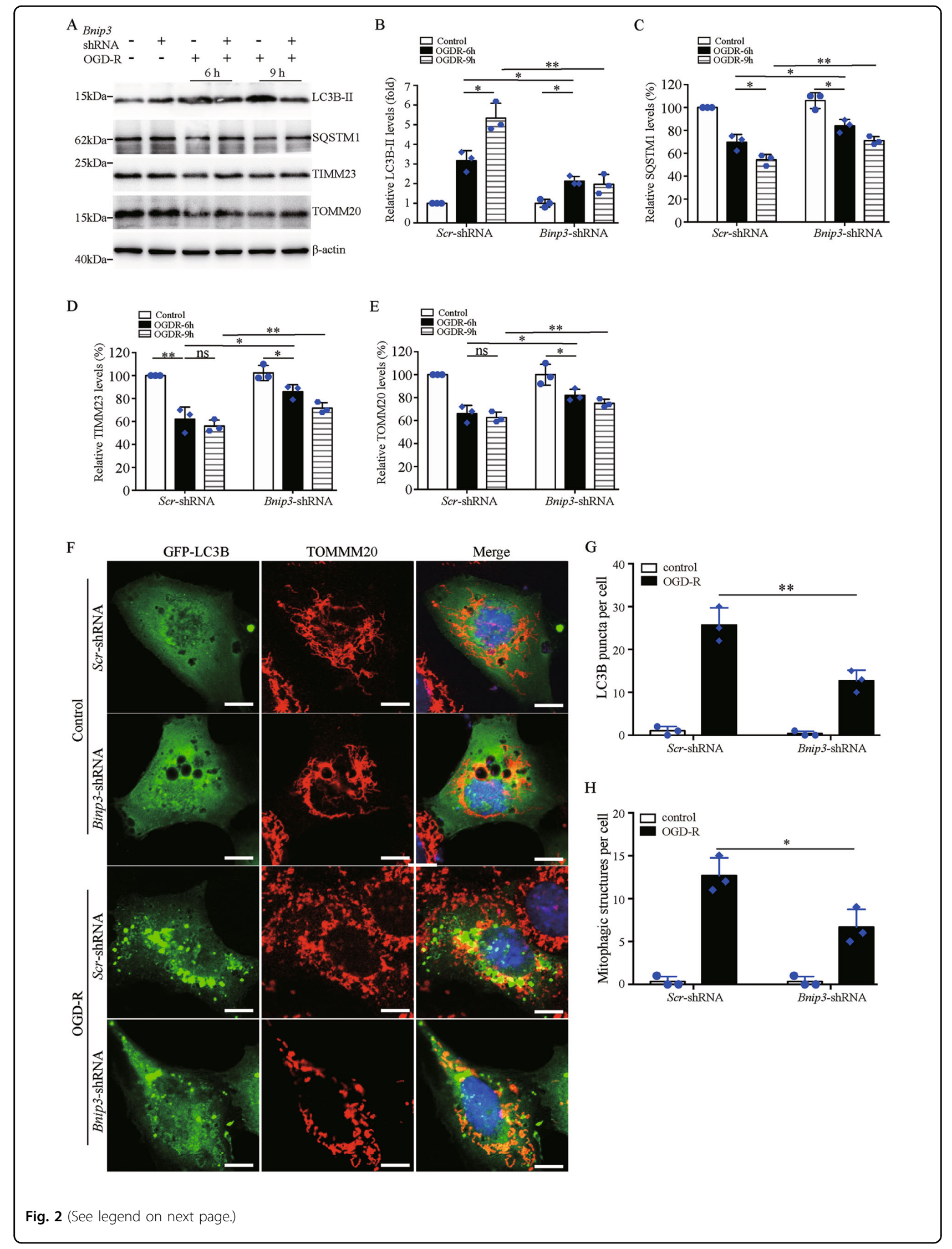


(see figure on previous page)

Fig. 2 Suppression of Bnip3 expression reduces OGD-R-induced mitophagy in BUMPT cells. a Representative blots. BUMPT cells stably expressing Scr-shRNA or Bnip3-shRNA were subjected to $2 \mathrm{~h}$ of OGD, followed by reperfusion for 6 or $9 \mathrm{~h}$. Whole-cell lysates were collected for immunoblot of LC3B-II, SQSTM1, TIMM23, TOMM20, and $\beta$-actin. b-e Densitometry of LC3B-II (b), SQSTM1 (c), TIMM23 (d), and TMOM20 (e) signals $(n=3)$. For densitometry, the signals of the target proteins were normalized to the $\beta$-actin signal of the same samples to determine the ratios. The ratios in sample of Scr-shRNA cells without OGD-R were arbitrarily set as 1 or 100\% in each blot. f Representative images of autophagosome and mitophagosome. Bar: $10 \mu \mathrm{m}$. BUMPT cells stably expressing Scr-shRNA or Bnip3-shRNA were transiently transfected with GFP-LC3B to label autophagosome. At $24 \mathrm{~h}$ after transfection, cells were subjected to $2 \mathrm{~h}$ of OGD, followed by $6 \mathrm{~h}$ of reperfusion. Cells cultured in regular medium and incubator were used as control. The cells were then fixed and stained with primary antibody against TOMM20 and corresponding fluorescent-labeled secondary antibody to label mitochondria. Nuclei were stained with DAPI (blue). $\mathbf{g}$ Quantification for LC3B puncta. The number of LC3B puncta in at least 30 cells from three different experiments was counted to indicate autophagosome formation. $\mathbf{h}$ Quantification for mitophagosome formation. LC3B puncta colocalizing with mitochondria in at least 30 cells from three different experiments were counted to indicate mitophagosome formation. Each symbol (circle and diamond) represents an independent experiment. Error bars: SEM, $n=3 .{ }^{*} P<0.05 ;{ }^{* *} p<0.01$; ns not significant

mice had a significantly higher renal tubular damage score than WT mice after renal IR (Fig. 3e). In line with the results of histological analysis, serum creatinine concentration in both $\mathrm{KO}$ and WT mice was low and comparable following sham operation (Fig. 3f), and was dramatically increased in both mice after renal IR. However, the concentration of serum creatinine in ischemic $\mathrm{KO}$ was significantly higher than in WT mice in the same experimental setting (Fig. 3f), indicating more severe renal failure in Bnip3-KO mice after renal IR. Moreover, renal IR induced significantly higher levels of kidney injury molecule 1 (KIM1), a biomarker of AKI, in Bnip3- KO mice than in WT mice (Fig. 3g, h).

The effect of Bnip3 deficiency on renal tubular cell apoptosis was also evaluated by TUNEL assay and staining of active cleaved caspase-3. As shown in Fig. 4a, Bnip3-KO and WT kidneys had few TUNEL-positive tubular cells following sham operation condition, and both had an increase of the positive cells after renal IR. Quantification analysis demonstrated that there were significantly more TUNEL-labeled tubular cells in Bnip3-KO kidneys than in WT kidneys after renal IR (Fig. 4b). Consistently, staining and quantification analysis demonstrated that renal IR resulted in significantly more active caspase 3 -positive tubular cells in Bnip3-KO mice than in WT mice (Fig. 4c, d). Collectively, these in vivo findings provided compelling evidence supporting a renoprotective role of BNIP3 in ischemic AKI.

Bnip3 deficiency increases renal inflammation after renal IR

Inflammation contributes critically to the initiation and extension of $\mathrm{AKI}^{28}$. We therefore evaluated renal inflammation by assessing renal infiltration of macrophages and neutrophils. As shown in Fig. 5a-c, renal IRinduced macrophage and neutrophil infiltration into kidneys in both Bnip3-KO and WT mice. Quantification analysis showed that ischemic Bnip3-KO mice had more macrophages (Fig. 5b) and neutrophils (Fig. 5c) infiltrating the kidney than ischemic WT mice. Moreover, quantitative real-time polymerase chain reaction (qRTPCR) analysis of the expression of proinflammatory cytokines interleukin-1 $\beta$ (IL-1b) and tumor necrosis factor- $\alpha$ (TNF- $\alpha)$ in kidney tissues demonstrated that renal IR induced significantly higher expression of these cytokines in Bnip3-KO mice than in WT mice (Fig. 5d, e). These results support that Bnip3-KO mice have much stronger interstitial inflammation than WT mice after renal IR.

\section{Bnip3 deficiency accumulates damaged mitochondria and increases ROS production following renal IR}

We examined the effect of Bnip3 deficiency on mitochondrial integrity in RPTCs by transmission electron microscopy (TEM) and morphometric analysis as previously described ${ }^{18,29}$. Under controlled condition, Bnip3$\mathrm{KO}$ and WT mice had tubular mitochondria in RPTCs (Fig. 6a). After renal IR, WT mice had an increase of small and round mitochondria, indicating mitochondrial fragmentation (Fig. 6a). Notably, loss of BNIP3 showed profound effects on mitochondrial structure, including the increase in mitochondrial fragmentation and swelling, vacuoles in the mitochondrial matrix, and loss of cristae. Quantification analysis of abnormal mitochondria that were swollen with evidence of severely disrupted cristae revealed that renal IR resulted in significantly more damaged mitochondria in RPTCs in Bnip3-KO mice than in WT mice (Fig. 6a, b). Collectively, these findings suggest that loss of Bnip3 accumulates damaged mitochondria in renal tubular cells following renal IR. Mitochondrial damage and dysfunction are associated with increased ROS production. Thus, we determined ROS levels within kidney tissues by dihydroethidium (DHE) staining. Under the condition of sham operation, both WT and KO mice had similarly low intensity of DHE signals in RPTCs (Fig. 6c). However, KO and WT kidneys showed a dramatic increase in the DHE signals in RPTCs after renal IR (Fig. 6c), and quantification analysis demonstrated that the $\mathrm{KO}$ mice had significantly stronger DHE signals than WT mice (Fig. 6d). These results of 


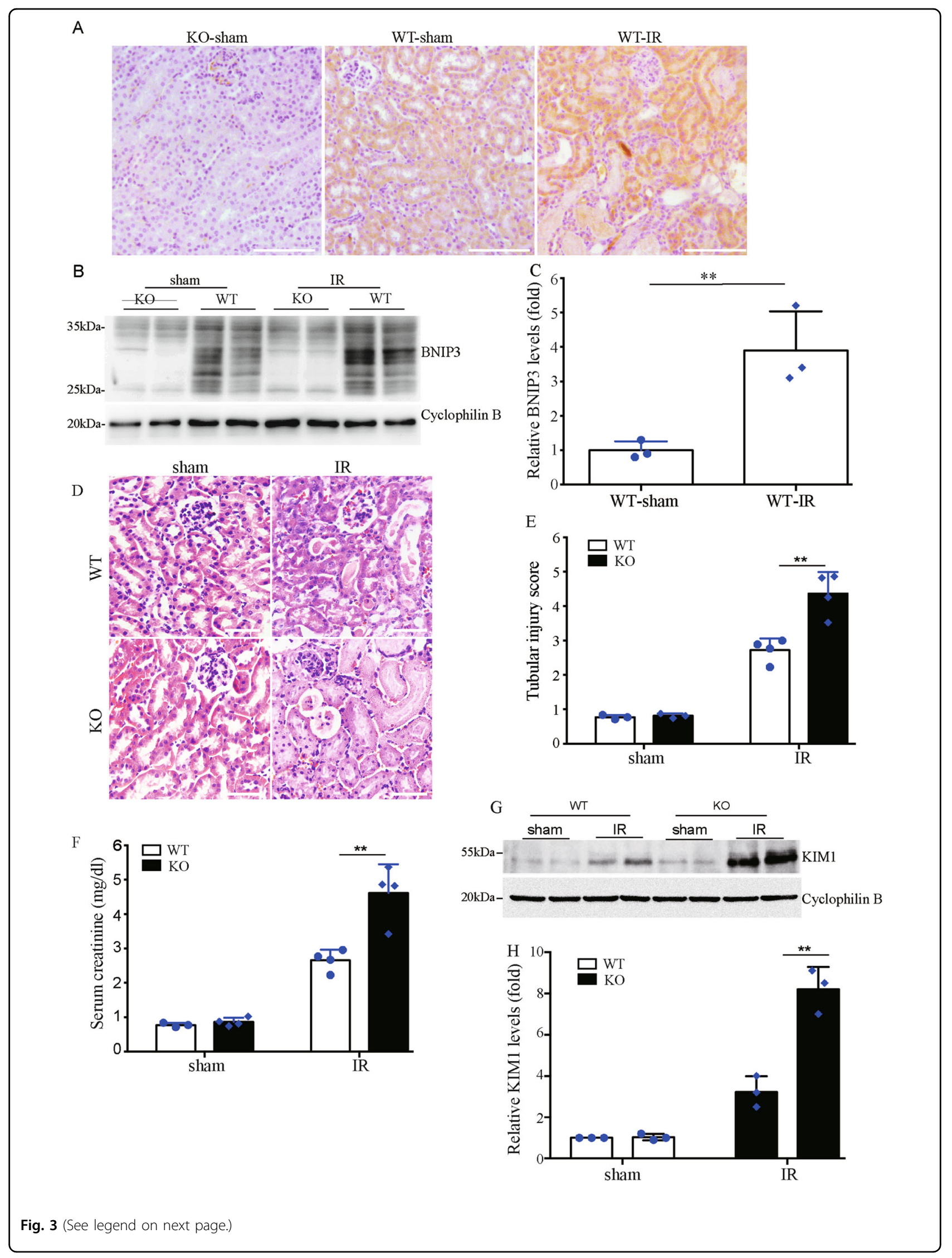


(see figure on previous page)

Fig. 3 Bnip3 deficiency exacerbates renal IR-induced kidney injury. Bnip3 deficiency (KO) mice and their wild-type littermates (WT) (male, 8 weeks old) were subjected to $30 \mathrm{~min}$ bilateral renal ischemia followed by $48 \mathrm{~h}$ of reperfusion (IR) or sham operation (sham). Kidney tissues were collected for histological and biochemical analysis. a Representative images of BNIP3 staining. b Representative immunoblot of BNIP3. c Densitometry of BNIP3 signals $(n=3)$. For densitometry, the BNIP3 signals were normalized to the cyclophilin B signal of the same samples to determine the ratios. d Representative images of hematoxylin-eosin staining. Bar: $100 \mu \mathrm{m}$. e Pathological score of tubular damage $(n=4)$. $\mathbf{f}$ Blood samples were collected for measurements of serum creatinine $(n=4)$. $\mathbf{g}$ Representative immunoblot of KIM1. $\mathbf{h}$ Densitometry of KIM1 signals $(n=4)$. The KIM1 signals were normalized to the cyclophilin B signal of the same samples to determine the ratios. The ratios of sham-operated WT were arbitrarily set as 1. Each symbol (circle and diamond) represents an individual mouse. Error bars: SEM. ${ }^{* *} p<0.01$

DHE staining are in line with the findings of more accumulation of damaged mitochondrial in RPTCs of Bnip3KO mice.

\section{Bnip3 deficiency reduces renal IR-induced mitophagy in vivo}

The marked accumulation of damaged mitochondria in RPTCs of ischemia-operated Bnip3-KO mice suggests possibly defective mitochondrial elimination. Therefore, we evaluated the effect of loss of Bnip3 on mitophagy in kidneys. Immunoblotting analysis showed an increase of LC3B-II and a concurrent decrease of SQSTM1 in WT kidney tissues after renal IR (Fig. $7 \mathrm{a}-\mathrm{c}$ ), and their changes were accompanied by a remarkable reduction of mitochondrial protein TIMM23 (Fig. 7a-d), suggesting mitophagy and mitochondrial clearance. Notably, the renal IRinduced changes in LC3B-II, SQSTM1, and TIMM23 in WT kidneys were partially but significantly reversed in ischemic Bnip3-KO kidney (Fig. 7a-d), suggesting an inhibitory effect of loss of BNIP3 on mitophagy. We further evaluated autophagosome and mitophagosome formation by immunofluorescence staining in kidney tissues using primary antibody against LC3B and mitochondrial protein cytochrome $c$ oxidase subunit 4I1 (COX4I1/ COXIV), respectively, and corresponding fluorescently labeled secondary antibodies. As shown in Fig. 7e, LC3B puncta were rarely observed in kidney tissues of shamoperated mice regardless of Bnip3 status, but were dramatically increased in kidney tissue of ischemic Bnip3-KO and WT mice. Of note, partial LC3B-labeled autophagosomes colocalized with the mitochondria, indicating the formation of mitophagosomes (Fig. 7e). Quantification analysis demonstrated that there was significantly less renal tubules with autophagosome and mitophagosome formation in Bnip3-KO mice compared to WT mice after renal IR (Fig. 7e-g), suggesting a reduction of mitophagy under the condition of Bnip3 deficiency. Further examination by using TEM confirmed autophagosome and mitophagosome formation in tubular cells after renal IR (Fig. 7h), which were rarely observed in kidney tissues from sham-operated mice. Taken together, these findings provide in vivo evidence that BNIP3 has a role in mitophagy regulation in renal tubular cells in ischemic AKI.

\section{Discussion}

The pathophysiological role of BNIP3 in AKI remains unknown. In the present study, we have provided substantial evidence supporting a renoprotective role of BNIP3 in renal tubular cells via regulating mitophagy. We demonstrates that BNIP3 is upregulated in RPTCs in both in vitro and in vivo models of ischemic AKI, and loss of BNIP3 aggravates ischemic injury. We provide further evidence that loss of BNIP3 reduces mitophagy, resulting in the accumulation of damaged mitochondria and ROS, increases of cell death, and enhancement inflammatory response in kidneys following renal IR. Thus, these findings indicate that enhancement of mitophagy may represent a promising novel therapeutic approach for AKI.

Upregulation of BNIP3 in RPTCs has been implicated in an experimental rat model of ischemic $\mathrm{AKI}^{20}$. However, the pathophysiological role of BNIP3 in AKI pathogenesis remains largely unknown. BNIP3 was initially identified as a pro-death protein ${ }^{22}$, and its pro-death function has been associated with the pathogenicity of cardiac diseases ${ }^{30-33}$. For instance, in animal models of cardiac ischemiainduced myocardial infarction, BNIP3 induction in ventricular myocytes occurred during early cardiac ischemia and persisted throughout reperfusion in an accompany with mitochondrial dysfunction and cardiomyocyte death $^{32}$. More importantly, inhibition of BNIP3 activity by gene deletion or expression of dominant-negative form of BNIP3 dramatically reduced myocardial apoptosis and myocardial injury after cardiac $I^{30,34}$, suggesting a pathogenic role of BNIP3 induction in cardiac infarction. On the other hand, emerging evidence also supports a cytoprotective role of BNIP3 in some disease conditions ${ }^{24-27}$. Our present study provides substantial evidence supporting a renoprotective role of BNIP3 in ischemic AKI, as evidenced by much more severe kidney structural and functional damage (Fig. 3d-h), more tubular cell death (Fig. 4), and stronger inflammation (Fig. 5) in the kidney of Bnip3-KO mice compared to their WT littermates after renal IR. The discrepancy concerning BNIP3 function in different tissues may suggest cell-typespecific functions of BNIP3. However, the underlying determinants of the pro-survival or pro-death functions of BNIP3 in different conditions remains poorly understood. 


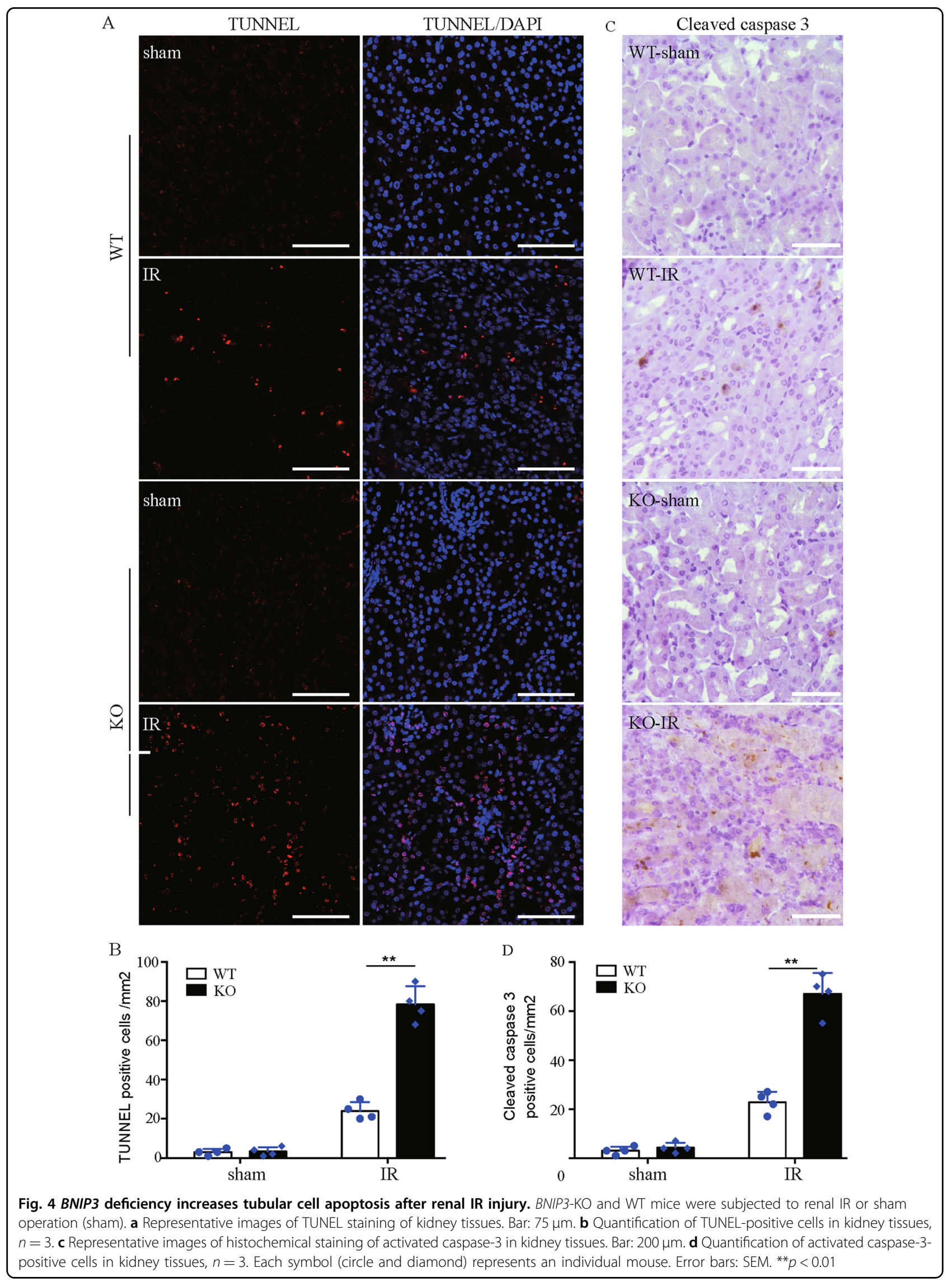




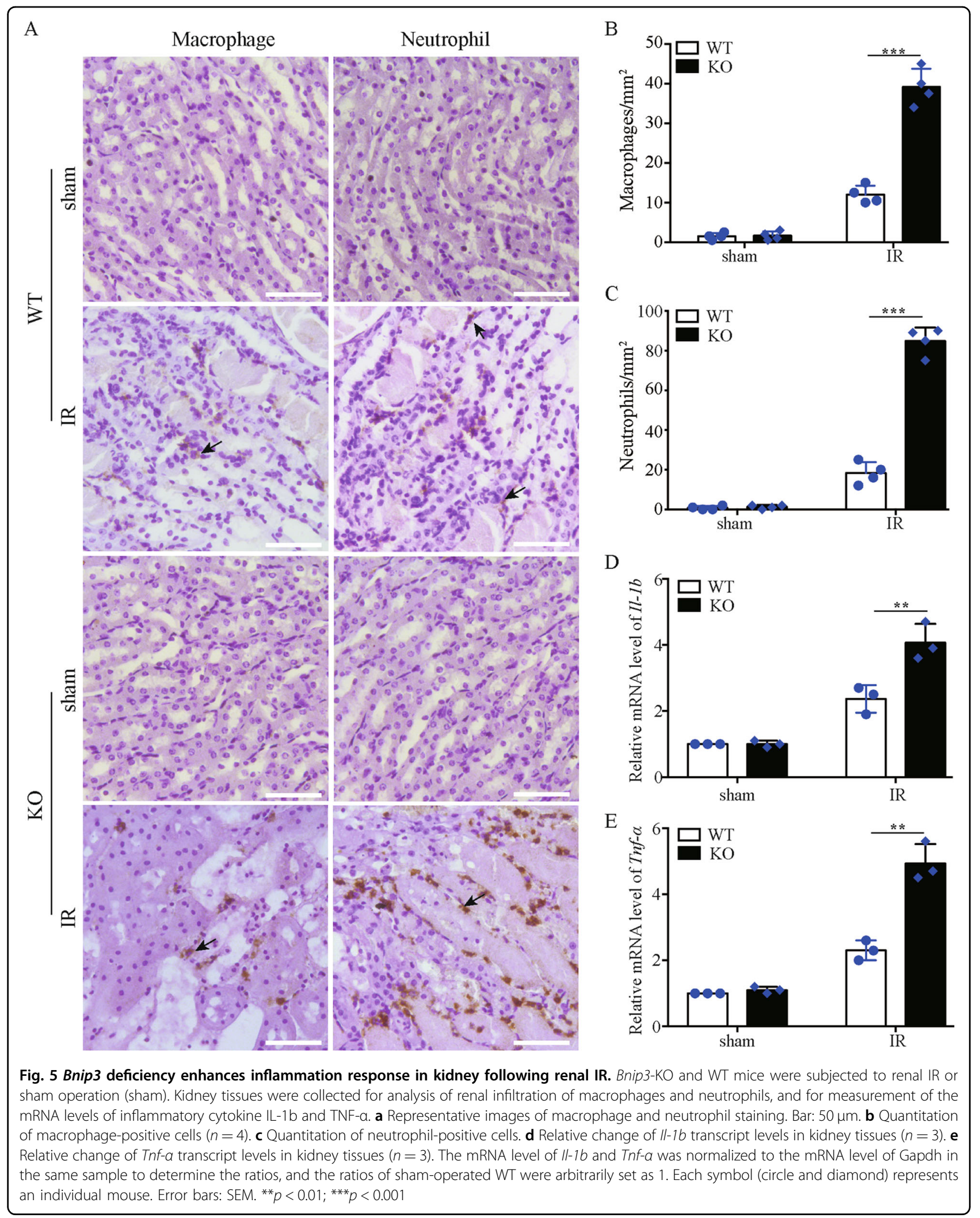




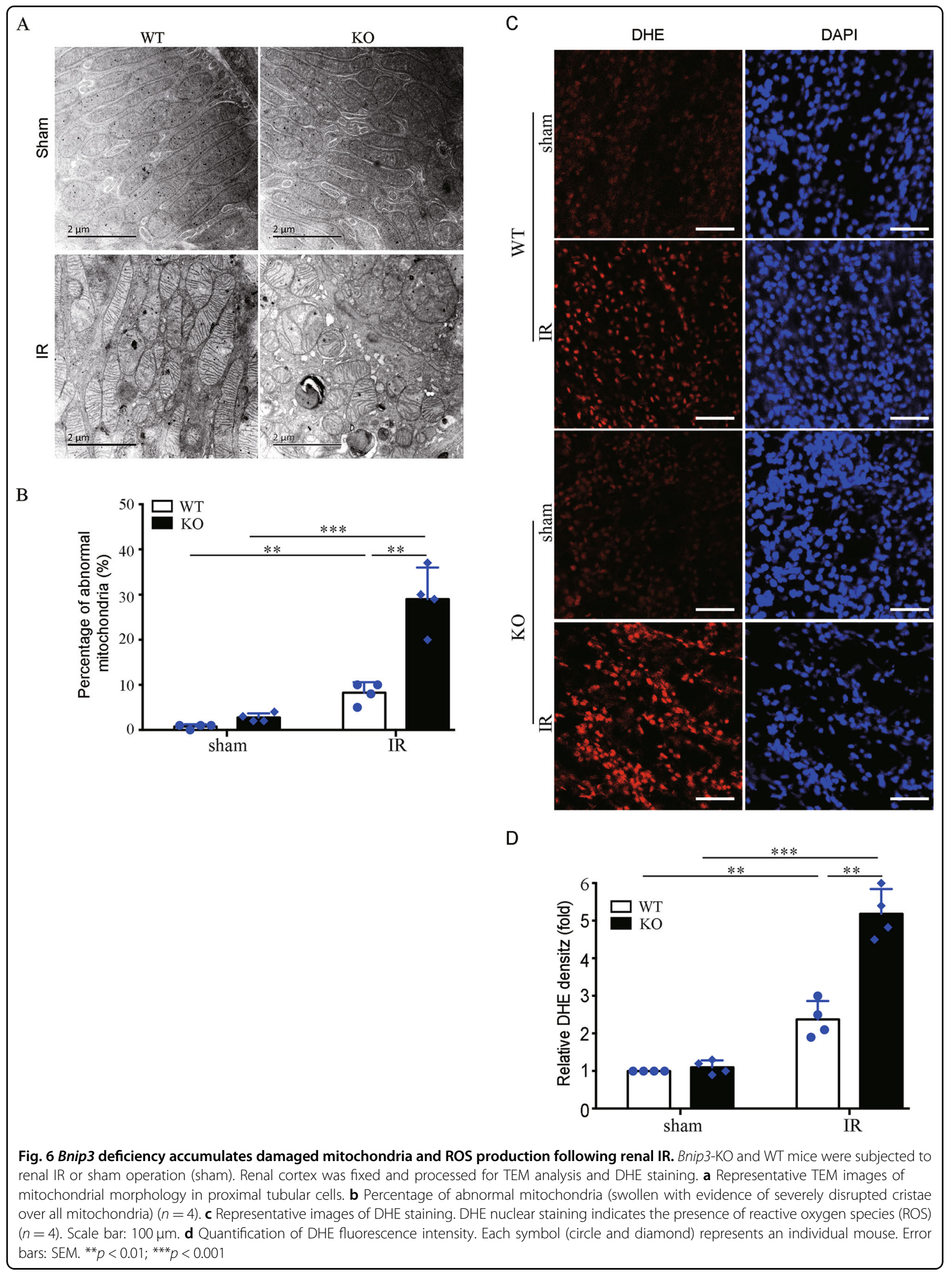




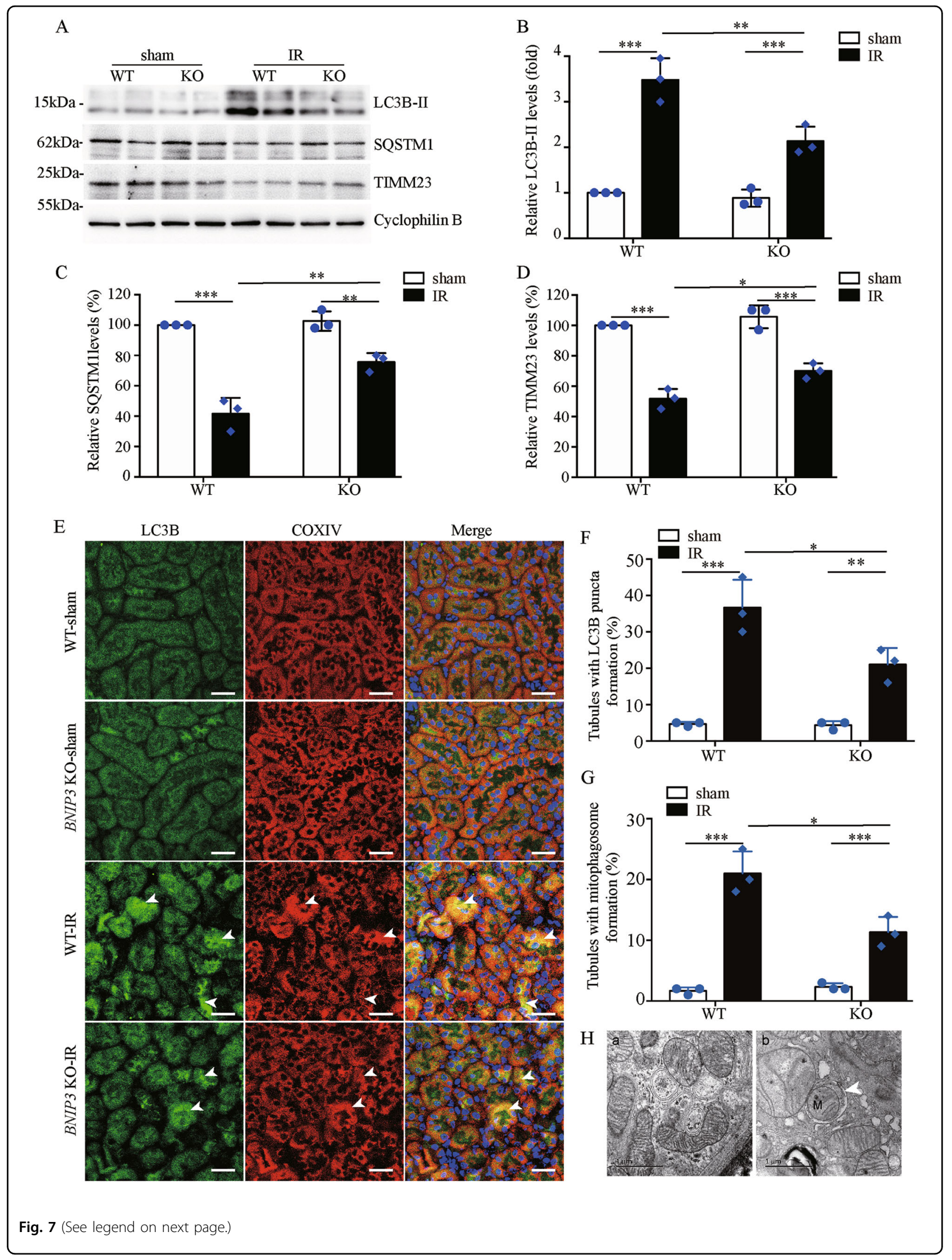


(see figure on previous page)

Fig. 7 Bnip3 deficiency suppresses renal IR-induced mitophagy. Bnip3-KO and WT mice were subjected to renal IR or sham operation (sham). Renal cortex was collected for immunoblot analyses of LC3B-I/II, SQSTM1, TIMM23, and cyclophilin B (loading control), immunofluorescence of LC3B and COXIV, and for transmission electronic microscopy (TEM) analysis. a Representative blots. b-d Densitometry of LC3B-II (b), SQSTM1 (c), and TIMM23 (d). For densitometry, the target proteins signals were normalized to the cyclophilin B signal of the same samples to determine the ratios, and the ratios of sham-operated WT were arbitrarily set as 1 or 100\%. e Representative images of immunofluorescence staining of LC3B (green) and COXIV (red) and DAPI (blue). $\mathbf{f}$ Percentage of renal tubules with LC3B puncta. $\mathbf{g}$ Percentage of renal tubules with mitophagosome formation (LC3B puncta colocalizes with mitochondria) in renal tubules. Bar: $50 \mu \mathrm{m}$. $\mathbf{h}$ TEM images of mitophagosome (pointed by arrowhead in the right panel) in renal proximal tubule cells after IR. Each symbol (circle and diamond) represents an individual mouse. Error bars: SEM, $n=3 .{ }^{*} P<0.05$; ${ }^{* *} p<0.01$; ${ }^{* * *} p<0.001$

BNIP3 has recently been recognized as a regulator of mitophagy ${ }^{24,25}$, but its pro-mitophagy function in kidney remains largely unknown. In present study, several lines of evidence supported an occurrence of BNIP3-mediated mitophagy in renal tubular cells in ischemic AKI, which includes: (1) upregulation of BNIP3 was associated with an increase of autophagy flux and mitochondrial proteins degradation, as well as mitophagosome formation in renal tubular cells following renal IR (Figs. 2 and 7); (2) all of these above changes were partially reversed by loss of BNIP3 (Figs. 2 and 7); (3) loss of BNIP3 led to the accumulation of damaged mitochondria following renal IR (Fig. 6). Of note, recent studies from us and others have demonstrated an induction of PINK1-PARK2 pathway of mitophagy in RPTCs during $\mathrm{AKI}^{17-19}$. Collectively, these findings strongly support an involvement of mitophagy in AKI pathogenesis, and, moreover, mitophagy in AKI may involve multiple pathways. Recent studies also provide evidence that there exists cross-talks between different mitophagy pathways. For instance, in cardiac myocytes, PARK2 translocation to mitochondria was demonstrated to be essential for BNIP3-mediated mitophagy ${ }^{35}$. More recently, Zhang et al. $^{24}$ provided evidence that BNIP3 binding to PINK1 suppressed PINK1 proteolytic cleavage and therefore increased the accumulation of intact PINK1 on the outer membrane of mitochondria, leading to increased PARK2 recruitment to the mitochondria and consequent mitophagy induction. Thus, it will be interesting to investigate whether BNIP3 and PINK1/PARKIN pathways of mitophagy act coordinately in regulating renal tubular cell mitophagy in AKI.

Mitophagy is a critical component of the mitochondrial quality control, which is critical for the maintenance of a healthy mitochondrial population and thereby cell viability. Under conditions when damaged mitochondria overwhelm the capacity of mitophagy, and/or mitophagy is impaired, damaged mitochondria accumulate, which may result in excessive ROS production, activation of cell death, and release of mitochondrial DAMPs. In consistent with previous reports, we verified that renal IR induced mitochondrial damage and increased ROS production in renal tubular cells, and, more importantly, these changes were aggravated within kidneys of Bnip3-KO mice in the same experiment (Fig. 6). It is conceivable that failing of BNIP3-mediated mitophagy expands renal IR-induced mitochondrial damage, which leads to further damage to the mitochondria and ultimately irreversible cell injury and cell death. Moreover, ROS, mitochondrial DAMPs, and various factors or contents released from necrotic tubular cells may stimulate robust inflammation to contribute tissue damage. In line with this notion, renal IR induced a stronger inflammatory response in the kidney of BNIP3-KO mice than in WT mice, as evidenced by significantly more macrophage and neutrophil infiltration into kidneys and higher expression of proinflammatory cytokines in these KO mice compared to WT mice (Fig. 5).

In summary, this study has provided substantial evidence for the activation of BNIP3-mediated mitophagy in RPTCs in ischemic AKI. We provided further evidence that BNIP3-mediated mitophagy has important role in mitochondrial quality control, tubular cell survival, and renal function during AKI. Notably, previous reports suggest that induction of PINK1/PARKIN pathways of mitophagy protects against AKI. As such, enhancing mitophagy may offer a novel therapeutic strategy for AKI.

\section{Material and methods \\ Cells, plasmids, and transfection}

BUMPTs were originally from Dr. Wilfred Lieberthal (Boston University School of Medicine) and maintained as described previously ${ }^{36}$. Bnip3-KD cells were generated by infection of BUMPT cells with Bnip3-shRNA-encoding lentivirus. Briefly, shRNAs specifically targeting mouse Bnip3 were synthesized by GenePharma (China), and then inserted into lentivirus vector PLKO.1 (Addgene plasmid \#8453). After viral packaging, the viruses encoding Bnip3 shRNA were harvested to infect BUMPT cells. Infected cells were selected with $2.5 \mathrm{mg} / \mathrm{ml}$ puromycin (SigmaAldrich) to generate stable cell line with Bnip3 KD. The KD efficiency was determined by qRT-PCR and immunoblotting analysis. The shRNA target sequences of Bnip3 were as follows: forward, 5'-CAGCCTCCGTCTCTA TTTA-3'; reverse, 5' -TACCAACAGAGCTGAAATA-3'. The GFP-LC3B plasmid was obtained from Addgene (\#11546). Transfections of BUMPT cells with plasmid DNAs were performed by using Lipofectamine 3000 
reagents (L3000008; Thermo Fisher Scientific) under the manufacturer's instruction.

\section{Antibodies and reagents}

Anti-SQSTM1 (5114), anti-COXIV (11967), and antiactivated caspase-3 (9664) were from Cell Signaling Technologies; anti-LC3B (NB100-2220) were from Novus Biologicals; anti-TIMM23 (sc-514463) and anti-TOMM20 (sc-11415) were purchased from Santa Cruz Biotechnology; anti-KIM1/HAVCR1 (hepatitis A virus cellular receptor 1) from R\&D Systems; anti-cyclophilin B, antimacrophage (RM0029-11H3), anti-neutrophil (NIMPR14), and anti-BNIP3 (ab109362, ab10433) were obtained from Abcam; anti- $\beta$-actin (A5316) was from SigmaAldrich. All secondary antibodies, DHE (D11347) and collagen I (A1048301), were purchased from Thermo Fisher Scientific, and TUNEL assay kit (12156792910) was obtained from Roche Life Science.

\section{Oxygen-glucose deprivation-reperfusion}

OGD-R in BUMPT cells was performed as previously described with modification ${ }^{37}$. Briefly, BUMPT cells were seeded in collagen I-coated plates at $24 \mathrm{~h}$ before treatment. Cells at an $\sim 95 \%$ confluence were rinsed once with glucosefree Dulbecco's modified Eagle's media (Thermo Fisher Scientific, 11966), and then maintained in both O2 and glucosefree medium. Cells were immediately placed in a sealed incubator chamber (Billups-Rothenburg, MIC-101), and then loaded with $\mathrm{N} 2$ gas for $10 \mathrm{~min}$ at $25 \mathrm{~L} / \mathrm{min}$. After incubation at $37^{\circ} \mathrm{C}$ for $2 \mathrm{~h}$, cells were transferred back to normal culture medium and regular incubator with oxygen for reperfusion. Cells cultured in normal medium and regular cell incubator with $21 \%$ oxygen were used as control.

\section{Animals and renal IR}

Animal experiments were conducted in accordance with a protocol approved by the Institutional Animal Care and Use Committee of the Second Xiangya Hospital of Central South University. C57BL/6 mice were purchased from SJA Laboratory Animal Corporation (Hunan, China). Bnip3-KO mice were previously described ${ }^{24}$. Mice were housed in a pathogen-free condition under cycles of 12:12-h light and dark with free access to food and water. Renal IR surgery in mice was performed as previously described $^{18}$. Briefly, bilateral renal pedicles were exposed for clamping to induce $30 \mathrm{~min}$ of ischemia, and the clamps were then released for reperfusion for $48 \mathrm{~h}$. Mice underwent the same operation without renal pedicle clamping were as sham control mice.

\section{Renal function and histopathology}

Serum creatinine was evaluated to determine renal functions with a kit from BioAssay Systems (DICT-500) as previously described ${ }^{18}$. To determine tissue damage, paraffin- embedded kidney tissue sections with a thickness of $4 \mu \mathrm{m}$ were stained with HE. Renal tubules with loss of brush border, tubular dilation and disruption, cast formation, and cell lysis were considered damaged. Tissue damage was evaluated by the percentage of damaged tubules: 0 , no damage; $1,<25 \%$; 2, 25-50\%; 3, 50-75\%; 4, >75\%.

\section{Immunohistochemical analysis}

Immunohistochemical staining was performed as described in our recent studies ${ }^{18}$. Briefly, paraffinembedded kidney sections were sequentially underwent deparaffinization, hydration, and antigen retrieval by incubation with $0.1 \mathrm{M}$ sodium citrate, $\mathrm{pH} 6.0$ at $100^{\circ} \mathrm{C}$. After subsequent incubation in $3 \% \mathrm{H}_{2} \mathrm{O}_{2}, 5 \%$ normal donkey serum and $0.1 \%$ Triton $\mathrm{X}-100$ to reduce nonspecific binding, tissue sections were exposed to 1:100 anti-BNIP3 (Abcam, Ab10433), 1:200 anti-cleaved caspase-3 (5A1E) (Cell Signaling, 9664), 1:100 anti-macrophage (Abcam, RM0029-11H3) or 1:100 antineutrophil (Abcam, NIMP-R14) at $4{ }^{\circ} \mathrm{C}$ overnight followed by exposure to horseradish peroxidase (HRP)conjugated secondary antibody for $1 \mathrm{~h}$ at room temperature. Signals of the antigen-antibody complexes were detected with a DAB Peroxidase Substrate Kit (Vector Laboratories) following the manufacturer's introduction. Sections were then counterstained with DAPI (4',6-diamidino-2-phenylindole) (Sigma-Aldrich, D9542). For quantification, 10-20 fields were randomly selected from each tissue section and the amounts of positive cells per $\mathrm{mm}^{2}$ was evaluated.

\section{TUNEL assay}

TUNEL assays were performed to evaluate cell apoptosis in kidney tissue and BUMPT cells using an In Situ Cell Death Detection Kit (Roche Applied Science, Indianapolis, IN) as described in our recent studies ${ }^{29,38}$. For quantification, the number of TUNEL-positive tubular cells in kidney sections in at least 10 optical fields in each tissue section from three different kidneys per group was counted, and the amount of TUNEL-positive cells per $\mathrm{mm}^{2}$ was evaluated. For quantification of TUNEL-positive BUMPT cells, at least 10 optical fields with $>200$ cells from three different experiments were examined in each condition to estimate the apoptosis percentage.

\section{Determination of ROS}

DHE staining of frozen kidney tissues were performed to elevated ROS levels as previously described ${ }^{18}$. Briefly, after harvest, kidney tissues were immediately placed in Tissue-Tek optimal cutting temperature compound (Sakura Finetek, 4583), followed by snap freezing in liquid nitrogen. Unfixed frozen kidney sections of $20 \mu \mathrm{m}$ thickness were incubated in $10 \mu \mathrm{M}$ DHE at $37^{\circ} \mathrm{C}$ for $30 \mathrm{~min}$ followed by counterstaining with DAPI (Sigma-Aldrich, 
D9542). Samples were imaged with confocal microscopy (Leica, TCS SP5). For quantification, the fluorescent density in the nuclei of proximal tubular cells within 10 random optical field from three different kidneys per group was determined with the ImageJ software.

\section{Transmission electron microscopy}

TEM analysis was performed as recently described ${ }^{29}$. Immediately after harvest, kidney tissues were sequentially fixed in paraformaldehyde and glutaraldehyde (Sigma-Aldrich, 340855), post fixation in osmium tetraoxide (Sigma-Aldrich, 201030), and dehydration in ethanol. Samples were then embedded in Epon (SigmaAldrich, 45345). Seventy-nanometer-thick tissue sections were stained with uranyl acetate (TED PELLA, 19481) and lead citrate (Sigma-Aldrich, 15326). Samples were imaged by using the TEM (Leica). For quantification of abnormal mitochondria that were swollen with severely disrupted cristae, at least 400 individual mitochondria from four different kidneys per group were counted. The percentage of abnormal mitochondrial over all mitochondria was analyzed to indicate the degree of mitochondrial damage.

\section{Quantitative real-time PCR}

Total RNA from cells and kidney tissues was extracted with TRIzol reagent (Thermo Fisher Scientific, 15596026) according to the manufacturer's instruction. Complementary DNA was synthesized using reverse transcription reagents (Thermo Fisher Scientific, N8080234). Quantitative real-time PCR was performed with the TB Green Premix Ex Taq II reagent (TaKaRa, RR820B) on LightCycler96 Real-Time PCR System (Roche Life Science). For quantification, the mRNA levels of target genes were normalized to the mRNA levels of Gapdh. Primers used were as follows: Bnip3-forward, 5'-GCTCCCAG ACACCACAAGAT-3' and reverse, 5'-TGAGAGTAG CTGTGCGCTTC-3'; Tnf- $\alpha$-forward, $5^{\prime}$-CAGGCGGTG CCTATGTCTC-3' and reverse, 5'-CGATCACCCCGAA GTTCAGTAG-3'; $I l-1 b$-forward, 5'-GAAATGCCACC TTTTGACAGTG-3' and reverse, 5'-CTGGATGCTCTCATCAGGACA-3'; Gapdh-forward, 5'-AGGTCGGT GTGAACGGATTTG-3' and Gapdh reverse, 5'-GGG GTCGTTGATGGCAACA-3'.

\section{Immunofluorescent staining}

Immunofluorescence staining in cultured cells was performed as previously described ${ }^{24}$. Briefly, cells were washed once with phosphate-buffered saline (PBS), followed by fixation with $4 \%$ paraformaldehyde and subsequent permeabilization with $0.1 \%$ Triton X-100. After being blocked with $5 \%$ bovine serum albumin, cells were probed with 1:100 anti-LC3B and 1:100 anti-COXIV, followed by exposure to Alexa-conjugated secondary antibodies. Samples were imaged using a confocal microscope (Leica, TCS SP5). For quantification, the number of LC3B puncta and LC3B puncta colocalizing with the mitochondria from at least 30 cells in three independent experiments were counted. For immunofluorescence staining in kidney tissues, parafilmembedded tissue sections underwent deparaffinization, rehydration, and antigen retrieval by incubation with $1 \mathrm{mM}$ EDTA (pH 8.0) at $95-100^{\circ} \mathrm{C}$ for $1 \mathrm{~h}$. After being subsequently incubated with $3 \% \mathrm{H}_{2} \mathrm{O}_{2}, 5 \%$ normal donkey serum, and $0.1 \%$ Triton $\mathrm{X}-100$. Sections were exposed to primary antibodies at $4{ }^{\circ} \mathrm{C}$ overnight and detected with Alexa-conjugated secondary antibodies. Samples were imaged using a confocal microscope (Leica, TCS SP5). For quantification, the number of LC3B puncta and LC3B puncta colocalizing with mitochondrial signals from 10 to 20 random fields $(\times 400)$ for each slide from three different kidneys was counted to indicate autophagosome and mitophagosome formation.

\section{Immunoblot analysis}

Cells or kidney tissues were washed with PBS buffer and then lysed in sodium dodecyl sulfate (SDS) sample buffer (63 mM Tris- $\mathrm{HCl}, 10 \%$ glycerol, and 2\% SDS) containing protease inhibitor cocktail (Sigma-Aldrich). Protein concentration was determined by the BCA method with reagents from Thermo Scientific. Equal amount of proteins from different groups were separated by electrophoresis. After transferring onto polyvinylidene difluoride membrane, the membrane was sequentially incubated with blocking buffer ( $5 \%$ skim milk), a primary antibody and a corresponding HRP-linked secondary antibody. The target protein was visualized using chemiluminescent substrate (Thermo Scientific). The protein band intensity was quantified by the ImageJ software (NIH).

\section{Statistical analysis}

Statistical analysis was conducted with the Prism software (GraphPad). Statistical differences in multiple groups were determined by multiple comparisons with analysis of variance, followed by Tukey's post tests. Differences between two groups were determined by twotailed unpaired or paired Student's $t$ test. Quantitative data were expressed as means \pm SEM.

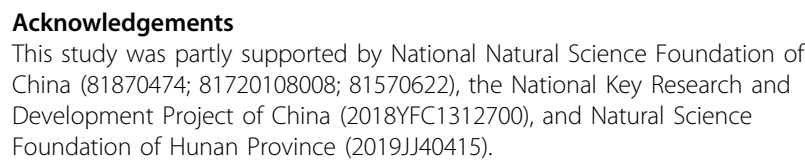
China (81870474; 81720108008; 81570622), the National Key Research and Development Project of China (2018YFC1312700), and Natural Science Foundation of Hunan Province (2019JJ40415).

\section{Author details}

'Department of Nephrology, The Second Xiangya Hospital, Central South University, Hunan Key Laboratory of Kidney Disease and Blood Purification, Changsha, Hunan, China. ${ }^{2}$ Institute of Molecular Precision Medicine, Xiangya Hospital and Center for Medical Genetics, Central South University, Changsha, Hunan, China. ${ }^{3}$ Department of Pathology and Laboratory Medicine, Indiana University School of Medicine, Indianapolis, IN, USA 


\section{Conflict of interest}

The authors declare that they have no conflict of interest.

\section{Publisher's note}

Springer Nature remains neutral with regard to jurisdictional claims in published maps and institutional affiliations.

Received: 10 June 2019 Revised: 6 August 2019 Accepted: 20 August 2019 Published online: 12 September 2019

\section{References}

1. Doyle, J. F. \& Forni, L. G. Acute kidney injury: short-term and long-term effects. Crit. Care 20, 188 (2016).

2. Venkatachalam, M. A., Weinberg, J. M., Kriz, W. \& Bidani, A. K. Failed tubule recovery, $\mathrm{AKI}-\mathrm{CKD}$ transition, and kidney disease progression. J. Am. Soc. Nephrol. 26, 1765-1776 (2015).

3. Zuk, A. \& Bonventre, J. V. Acute kidney injury. Annu. Rev. Med. 67, 293-307 (2016).

4. Wei, Q. et al. MicroRNA-668 represses MTP18 to preserve mitochondrial dynamics in ischemic acute kidney injury. J. Clin. Invest. 128, 5448-5464 (2018).

5. Bhargava, P. \& Schnellmann, R. G. Mitochondrial energetics in the kidney. Nat. Rev. Nephrol. 13, 629-646 (2017).

6. Parikh, S. M. et al. Mitochondrial function and disturbances in the septic kidney. Semin. Nephrol. 35, 108-119 (2015).

7. Lan, R. et al. Mitochondrial pathology and glycolytic shift during proximal tubule atrophy after ischemic AKI. J. Am. Soc. Nephrol. 27, 3356-3367 (2016).

8. Feldkamp, T., Kribben, A. \& Weinberg, J. M. F1FO-ATPase activity and ATP dependence of mitochondrial energization in proximal tubules after hypoxia/ reoxygenation. J. Am. Soc. Nephrol. 16, 1742-1751 (2005).

9. Guo, Y. et al. MicroRNA-709 mediates acute tubular injury through effects on mitochondrial function. J. Am. Soc. Nephrol. 29, 449-461 (2018).

10. Jesinkey, S. R. et al. Formoterol restores mitochondrial and renal function after ischemia-reperfusion injury. J. Am. Soc. Nephrol. 25, 1157-1162 (2014).

11. Gibbs, W. S. et al. 5-HT1F receptor regulates mitochondrial homeostasis and its loss potentiates acute kidney injury and impairs renal recovery. Am. J. Physiol. Ren. Physiol. 315, F1119-F1128 (2018).

12. Lynch, M. R. et al. TFEB-driven lysosomal biogenesis is pivotal for PGC1alphadependent renal stress resistance. JCl Insight 5, e126749 (2019).

13. Tran, M. T. et al. PGC1alpha drives NAD biosynthesis linking oxidative metabolism to renal protection. Nature 531, 528-532 (2016).

14. Tang, C., He, L., Liu, J. \& Dong, Z. Mitophagy: basic mechanism and potential role in kidney diseases. Kidney Dis. (Basel). 1, 71-79 (2015).

15. Nguyen, T. N., Padman, B. S. \& Lazarou, M. Deciphering the molecular signals of PINK1/Parkin mitophagy. Trends Cell Biol. 26, 733-744 (2016).

16. Liu, L., Sakakibara, K., Chen, Q. \& Okamoto, K. Receptor-mediated mitophagy in yeast and mammalian systems. Cell Res. 24, 787-795 (2014).

17. Wang, Y. et al. PINK1/Parkin-mediated mitophagy is activated in cisplatin nephrotoxicity to protect against kidney injury. Cell Death Dis. 9, 1113 (2018).

18. Tang, $C$. et al. PINK1-PRKN/PARK2 pathway of mitophagy is activated to protect against renal ischemia-reperfusion injury. Autophagy 14,880-897 (2018).
19. Zhao, C. et al. Pink1/Parkin-mediated mitophagy play a protective role in cisplatin induced renal tubular epithelial cells injury. Exp. Cell Res. 350, 390-397 (2017).

20. Ishihara, M. et al. Sestrin-2 and BNIP3 regulate autophagy and mitophagy in renal tubular cells in acute kidney injury. Am. J. Physiol. Ren. Physiol. 305, F495-F509 (2013).

21. Zhao, C. et al. Drp1-dependent mitophagy protects against cisplatin-induced apoptosis of renal tubular epithelial cells by improving mitochondrial function. Oncotarget 8, 20988-21000 (2017).

22. Kale, J., Osterlund, E. J. \& Andrews, D. W. BCL-2 family proteins: changing partners in the dance towards death. Cell Death Differ. 25, 65-80 (2018).

23. Burton, T. R. \& Gibson, S. B. The role of Bcl-2 family member BNIP3 in cell death and disease: NIPping at the heels of cell death. Cell Death Differ. 16, 515-523 (2009).

24. Zhang, T. et al. BNIP3 protein suppresses PINK1 kinase proteolytic cleavage to promote mitophagy. J. Biol. Chem. 291, 21616-21629 (2016).

25. O'Sullivan, T. E., Johnson, L. R., Kang, H. H. \& Sun, J. C. BNIP3- and BNIP3Lmediated mitophagy promotes the generation of natural killer cell memory. Immunity 43, 331-342 (2015).

26. Glick, D. et al. BNip3 regulates mitochondrial function and lipid metabolism in the liver. Mol. Cell. Biol. 32, 2570-2584 (2012).

27. Li, R. et al. Therapeutic effect of Sirtuin 3 on ameliorating nonalcoholic fatty liver disease: the role of the ERK-CREB pathway and Bnip3-mediated mitophagy. Redox Biol. 18, 229-243 (2018).

28. Akcay, A., Nguyen, Q. \& Edelstein, C. L. Mediators of inflammation in acute kidney injury. Mediators Inflamm. 2009, 137072 (2009).

29. Brooks, C., Wei, Q., Cho, S. G. \& Dong, Z. Regulation of mitochondrial dynamics in acute kidney injury in cell culture and rodent models. J. Clin. Invest. 119, 1275-1285 (2009).

30. Diwan, A. et al. Inhibition of ischemic cardiomyocyte apoptosis through targeted ablation of Bnip3 restrains postinfarction remodeling in mice. J. Clin. Invest. 117, 2825-2833 (2007).

31. Regula, K. M., Ens, K. \& Kirshenbaum, L. A. Inducible expression of BNIP3 provokes mitochondrial defects and hypoxia-mediated cell death of ventricular myocytes. Circ. Res. 91, 226-231 (2002).

32. Chaanine, A. H. et al. Potential role of BNIP3 in cardiac remodeling, myocardial stiffness, and endoplasmic reticulum: mitochondrial calcium homeostasis in diastolic and systolic heart failure. Circ. Heart Fail. 6, 572-583 (2013).

33. Chen-Scarabelli, C. et al. The role and modulation of autophagy in experimental models of myocardial ischemia-reperfusion injury. J. Geriatr. Cardiol. 11, 338-348 (2014).

34. Hamacher-Brady, A. et al. Response to myocardial ischemia/reperfusion injury involves Bnip3 and autophagy. Cell Death Differ. 14, 146-157 (2007).

35. Lee, Y., Lee, H. Y., Hanna, R. A. \& Gustafsson, A. B. Mitochondrial autophagy by Bnip3 involves Drp1-mediated mitochondrial fission and recruitment of Parkin in cardiac myocytes. Am. J. Physiol. Heart Circ. Physiol. 301, H1924-H1931 (2011).

36. Livingston, M. J. et al. Persistent activation of autophagy in kidney tubular cells promotes renal interstitial fibrosis during unilateral ureteral obstruction. Autophagy 12, 976-998 (2016).

37. Zhang, $X$. et al. Cerebral ischemia-reperfusion-induced autophagy protects against neuronal injury by mitochondrial clearance. Autophagy 9, 1321-1333 (2013).

38. Jiang, M., Liu, K., Luo, J. \& Dong, Z. Autophagy is a renoprotective mechanism during in vitro hypoxia and in vivo ischemia-reperfusion injury. Am. J. Pathol. 176, 1181-1192 (2010). 